\title{
Investigation of the Clinical, Radiological and Biological Factors Associated with Disease Progression, Phenotypes and Endotypes of COPD in China (COMPASS): study design, protocol and rationale
}

\author{
Zhenyu Liang ${ }^{1}$, Nanshan Zhong ${ }^{1}$, Rongchang $\mathrm{Chen}^{2}$, Qianli $\mathrm{Ma}^{3}$, Yongchang Sun ${ }^{4}$, Fuqiang $\mathrm{Wen}^{5}$, \\ Ruth Tal-Singer $\mathbb{1}^{6,10}$, Bruce E. Miller $\mathbb{D}^{6,10}$, Julie Yates ${ }^{7,10}$, Jie Song ${ }^{8}$, Chris Compton ${ }^{9}$, Beulah $\mathrm{Ji}^{8}$, Li $\mathrm{Wu}^{8}$, \\ Yang Yang ${ }^{8}$, Paul Jones $\mathbb{C}^{9}$ and Jinping Zheng ${ }^{1}$
}

\begin{abstract}
${ }^{1}$ State Key Laboratory of Respiratory Disease and National Clinical Research Center of Respiratory Disease, the First Affiliated Hospital of Guangzhou Medical University, Guangzhou Medical University, Guangzhou, China. ${ }^{2}$ Pulmonary and Critical Care Dept, Shenzhen Institute of Respiratory Diseases, First Affiliated Hospital of South University of Science and Technology of China (Shenzhen People's Hospital), Shenzhen, Guangdong, China. ${ }^{3}$ Chronic Respiratory Disease Care Centre, The North Kuanren General Hospital, Chongqing, China. ${ }^{4}$ Dept of Respiratory and Critical Care Medicine, Peking University Third Hospital, Beijing, China. ${ }^{5}$ Division of Pulmonary Diseases, State Key Laboratory of Biotherapy of China, and Dept of Respiratory Medicine, West China Hospital of Sichuan University, Chengdu, China. ${ }^{6}$ GlaxoSmithKline, Collegeville, PA, USA. ${ }^{7}$ GlaxoSmithKline, Research Triangle Park, NC, USA. ${ }^{8}$ GlaxoSmithKline, Research and Development, Shanghai, China. ${ }^{9}$ GlaxoSmithKline, Brentford, UK. ${ }^{10}$ No longer employees of GSK but were during the design of this study.
\end{abstract}

Corresponding author: Paul Jones (pjones@sgul.ac.uk)

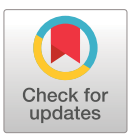

Copyight authors 202

This version is distributed under the terms of the Creative Commons Attribution NonCommercial Licence 4.0. For commercial reproduction rights and permissions contact permissions@ersnet.org

Received: 18 March 2021 Accepted: 24 June 2021

\section{○@®@}

Shareable abstract (@ERSpublications)

COMPASS, a prospective, multicentre, observational study of Chinese patients with COPD, will characterise stable and exacerbation phenotypes/endotypes, treatment pathways and HRU, and investigate COPD progression biomarkers' relevance to these patients https://bit.ly/3dylpf1

Cite this article as: Liang Z, Zhong N, Chen R, et al. Investigation of the Clinical, Radiological and Biological Factors Associated with Disease Progression, Phenotypes and Endotypes of COPD in China (COMPASS): study design, protocol and rationale. ERJ Open Res 2021; 7: 00201-2021 [DOI: 10.1183/ 23120541.00201-2021].

\section{Abstract}

COPD is heterogeneous, and its presentation varies between countries. The major COPD cohort studies have only been performed in Western populations; the disease is not well characterised in other regions. The COMPASS (Investigation of the Clinical, Radiological and Biological Factors, Humanistic and Healthcare Utilisation Burden Associated with Disease Progression, Phenotypes and Endotypes of COPD in China; NCT04853225) is a prospective, 2.5-year-long, multi-centre, longitudinal, observational study with three aims: 1) to characterise stable and exacerbation phenotypes/endotypes in terms of clinical characteristics, blood and sputum biomarkers, lung microbiome and lung imaging; 2) to understand the relevance of markers of COPD disease progression identified in Western cohorts to Chinese patients; and 3) to characterise treatment pathways and healthcare resource utilisation. COMPASS will recruit 2000 participants, of which 1700 will be in Global Initiative for Chronic Obstructive Lung Disease (GOLD) Grades I-IV (n=700, 700, 200 and 100, respectively), 180 participants with chronic bronchitis without airflow limitation and 120 never-smoker healthy controls. Study visits will be at baseline, 6, 18 and 30 months and at exacerbation. Assessments include lung function, exacerbation frequency, health status, blood biomarkers and, in a sub-cohort of 400 patients, chest high-resolution computed tomography, additional blood and sputum biomarkers, airway micro-, viral- and myco-biome, and physical activity. COMPASS will establish a unique clinical and biological dataset in a well-characterised cohort of individuals with COPD in China, with a particular focus on milder patients. As the first study of its kind attempting to understand the disease in an Asian setting, it will provide valuable insights into regional and ethnic differences in COPD. 
Introduction

COPD is one of the leading causes of morbidity and mortality resulting in significant humanistic and economic burden to patients, healthcare systems and societies worldwide. It is the third leading cause of death globally, and in 2019 there were 3.23 million deaths due to COPD [1]. COPD prevalence in China has been increasing significantly. A national screening survey, conducted from 2002 to 2004, reported that the overall prevalence of spirometry-defined COPD among people over 40 years old was $8.2 \%$ [2]. The recently published China Pulmonary Health Study (2018) suggested that the prevalence of COPD among Chinese over 40 years old had increased to 13.7\% [3]. Although COPD management in China has greatly improved over the past decades, many challenges persist. One of them is a substantial under-diagnosis: in a 2007 study, up to 65\% of people with airflow limitation pattern compatible with COPD did not have a clinical diagnosis of COPD [2]. Furthermore, most individuals diagnosed with COPD (64.7\%) exhibited a significant symptom burden, defined as at least one respiratory symptom (cough, phlegm, wheezing and breathlessness), regardless of treatment [2]. Its health status impact, as judged by COPD Assessment Test (CAT) score, also appears to be higher in China than in the West. In one study of over 6000 patients across China, the mean score was 24, compared to under 20 commonly reported in Europe [4].

COPD is a heterogeneous and complex condition displaying a range of phenotypes and endotypes with distinct underlying mechanisms, different clinical outcomes, varying patterns of progression, prognostic characteristics and different responses to treatment. The heterogeneity reflects the fact that not all COPD components are present in all patients and the complexity is due to dynamic and nonlinear interactions between the components that are present [5]. This indicates the need for personalised management, for example using a treatable traits approach to assessment, classification and management of COPD [6, 7].

Three large observational studies - Evaluation of COPD Longitudinally to Identify Predictive Surrogate End-points (ECLIPSE) [8], Genetic Epidemiology of COPD (COPDGene) Study [9], and Subpopulations and Intermediate Outcomes in COPD Study (SPIROMICS) [10] - were designed to address the complexity and heterogeneity of COPD, but they were all conducted in North America and Europe. A fourth study in individuals with obstructive lung disease (NOVELTY) is ongoing and includes patients in Asia. In contrast to the other three studies, it includes individuals with asthma as well as COPD, but does not have the same comprehensive range of in-depth assessment [11] (e.g. sputum biomarkers). The absence of studies in Asia is a limitation, not only locally within the region, but also globally in terms of understanding the full picture of the disease. For example, lung growth as measured by forced vital capacity (FVC) is related to a country's gross national income per capita [12], which is important with the increasing recognition that failure of full lung growth in childhood and early adulthood is a cause of COPD [13]. In addition, there may be substantial differences between countries in different continents in terms of respiratory symptoms and comorbid respiratory conditions [14], since significant racial differences have been reported between Korea and the USA in the pattern of extrapulmonary comorbidities [15]. Age-standardised disability-adjusted life years due to COPD vary quite widely between the USA and China and even within Europe [16]. Pollution is an important factor in COPD, and, setting aside the well-known effects of industrial pollution, levels of death due to indoor air pollution are much higher in China and Asia than in Europe and the USA [17]. Taken together with high levels of biomass fuel exposure for a significant part of the lives of currently middle-aged people before the relatively recent mass urbanisation that has taken place in China, these factors may result in a higher proportion of non-cigarette-smoking-related COPD.

COPD patients in China show significant differences when compared with Western patients. In the Asian cohort study [18] of the Tiotropium Safety and Performance In Respimat (TIOSPIR) trial [19], ZHONG et al. [18] demonstrated that Asian COPD patients had significantly lower body mass index (BMI), had a higher proportion of males, less cardiovascular disease and fewer but more severe exacerbations than those in the West. With an ageing population, persistent air pollution and significant biomass fuel exposure in China, the humanistic and economic burden of COPD is expected to increase and will require more healthcare resources in the future. COPD has been identified as a key disease of focus, and its appropriate management is a healthcare priority in China. To inform clinical practice and aid public health resource allocation, it is important to gain a better understanding of disease progression and clinical management of COPD in China. In particular it is important to understand the characteristics of the disease earlier in the evolution of the disease and in patients with milder airflow limitation, because this is where disease-modifying strategies will have greatest long-term benefit.

The COMPASS study (GlaxoSmithKline study Number 208630, ClinicalTrials.gov identifier: NCT04853225) is a 2.5-year longitudinal study with the overall objective of expanding understanding of the pattern of COPD disease phenotypes/endotypes, clinical, humanistic, and healthcare utilisation burden, 
disease progression and management of COPD in China, especially in patients with mild-to-moderate COPD. This article describes the purpose, design and objectives of this study.

\section{Methods}

\section{Study objective}

The key objectives of COMPASS are to: 1) characterise stable disease and exacerbation phenotypes/ endotypes in China through assessment of clinical characteristics, blood biomarkers, lung microbiome and radiological features of COPD; 2) understand the relevance of predictors of COPD disease progression identified in Western cohorts to Chinese patients; 3) characterise treatment pathways and healthcare resource utilisation and costs in COPD; 4) explore the utility of integrated digital data collected from multiple sources in the assessment and management of COPD patients.

\section{Study design}

COMPASS is a prospective, longitudinal 2.5-year multi-centre, non-drug interventional, observational study being conducted at 41 centres in Guangdong and Fujian, China. It will enrol 2000 participants into three strata (never-smoker healthy participants, chronic bronchitis without airflow limitation and COPD current or former smokers or never-smokers) as shown in figure 1. A sub-cohort with 400 participants will be studied in greater detail (figure 1).

\section{Study participants}

Inclusion criteria are participants must be $40-80$ years of age at baseline for the main cohort, have a smoking history of $<1$ pack-year (for never-smoking COPD participants, chronic bronchitis and healthy participants) or $\geqslant 1$ pack-year (smoking and ex-smoking COPD and chronic bronchitis) and meet lung function criteria as specified in table 1. Major exclusion criteria are respiratory disorders other than COPD (e.g. lung cancer, sarcoidosis, active tuberculosis, lung fibrosis, severe bronchiectasis, cystic fibrosis and alpha-1 antitrypsin deficiency). A current primary diagnosis of asthma is an exclusion criterion but individuals with a primary diagnosis of COPD but who also have had asthma can be included. Previous major lung surgery (e.g. lobectomy, lung reduction, lung transplant) is an exclusion criterion, as is a diagnosis of any cancer: current and within the last 5 years (patients in remission for $\geqslant 5$ years could be included). Table 1 contains the inclusion criteria. An exacerbation is defined as the occurrence of an episode of increased cough (with or without phlegm), shortness of breath or chest symptoms (chest discomfort or tightness) which lasted $48 \mathrm{~h}$ or more and interfered with the subject's usual activities. It is based on a definition used in the BOLD and CANCOLD surveys that also included mild patients [20, 21] To minimise the risk of ionising radiation as a consequence of repeated exposure to low-dose computed tomography scans of the chest, a minimum age of 50 years was selected for the sub-cohort. The study was approved by the ethics committee of each research centre and Human Genetic Resource Administration of China (HGRAC).

\section{Baseline and follow-up assessments}

There will be four on-site visits - baseline, 6 months, 18 months and 30 months - to collect concurrent medications, smoking status, spirometry, CAT score, healthcare resource utilisation/cost, etc. Participants will also receive trimonthly follow-up calls to assess CAT score, medication/treatment and exacerbation events. At baseline and 30 months biomarkers including the following will be collected: fibrinogen [22]/ high-sensitivity C-reactive protein (hsCRP)/blood cell count and blood biomarkers (inclusive of serum sRAGE, CC16, HbA1c and IP-10; sub-cohort only). In the sub-cohort, sputum cytology, sputum microbiome and chest high-resolution computed tomography (HRCT) scan (at maximum inspiration and expiration) will be performed.

\section{Outcome measurements}

End-points measured in COMPASS include rate of decline in forced expiratory volume in $1 \mathrm{~s}\left(\mathrm{FEV}_{1}\right)$, rate of moderate/severe exacerbations, change in health status scores over time, clinically important deterioration composite outcome, rate of decline in lung density change from baseline in airway diameter (quantified by HRCT; sub-cohort only), mortality and blood biomarker, etc. The key end-points are listed in figure 2.

\section{Airway micro-, viral- and myco-biome}

The airway microbial community (microbiome) is a complex mixture of organisms [23]. In COPD, airway microbial dysbiosis (change in microbiome diversity and composition) is associated with disease severity and exacerbations [24-26]. Such studies have almost exclusively assessed patients in North America and Europe. Although studies of airway microbiome are now being performed in Asia [15], there is very limited information on the composition of the airway microbiome and host-microbiome interactions in 


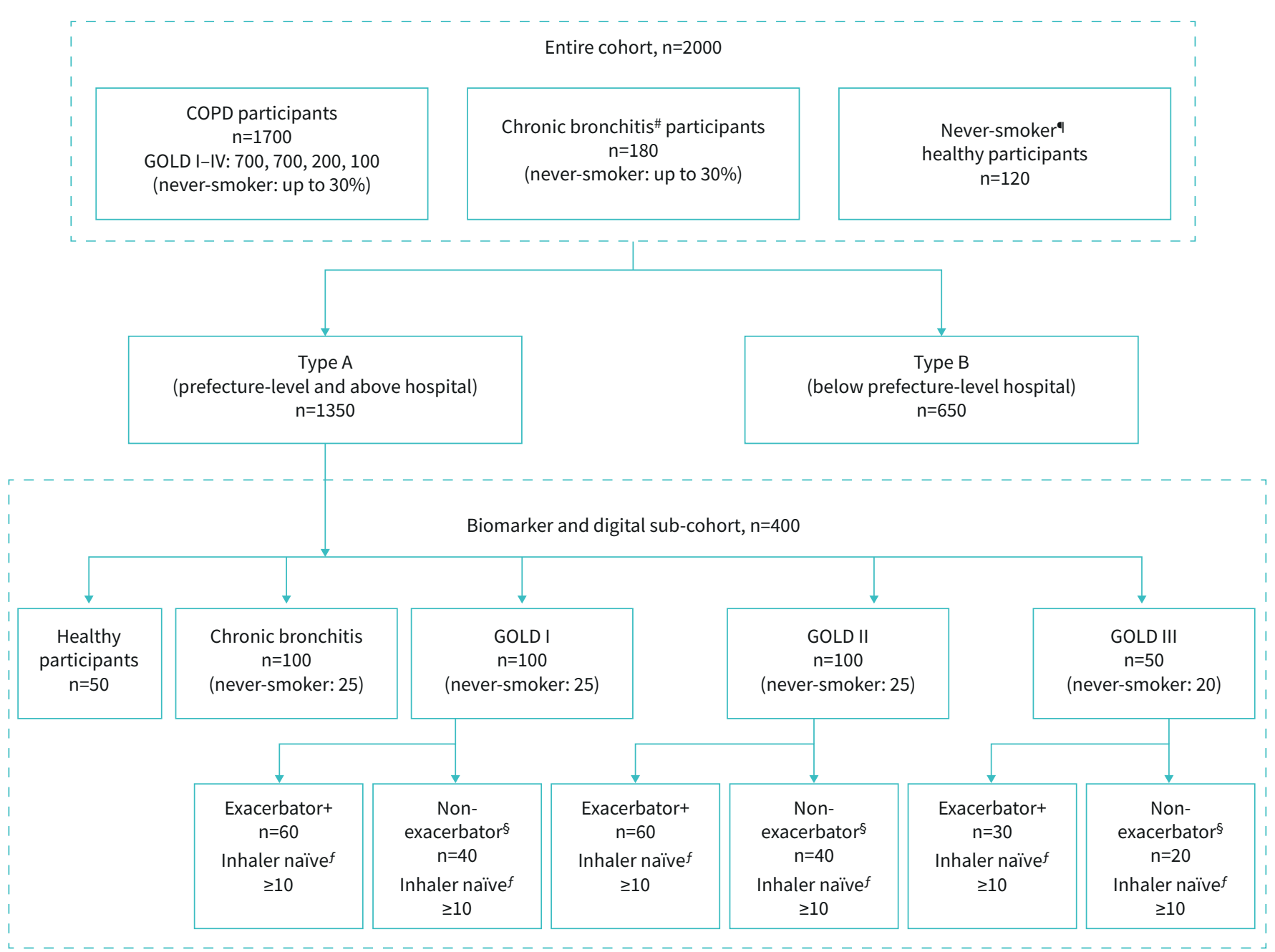

FIGURE 1 COMPASS enrolment strata. Type A hospitals include all hospitals at prefecture level or higher; Type B hospitals include all hospitals below the prefecture level, including community hospitals. ": chronic bronchitis defined by at least 3 months of cough and phlegm in a year in the past 2 years and normal spirometry. ": never-smokers are defined as a lifetime exposure of $<1$ pack-year. ${ }^{+}$: exacerbator is a patient who has one or more exacerbations in the 2 years before recruitment (see text). ${ }^{\S}$ : non-exacerbator is defined as a patient who has not had an exacerbation in the 2 years before entry into the study. ${ }^{f}$ : inhaled maintenance treatment naïve patients are defined as not being treated in at least prior 12 months with any long-acting inhaled medications inclusive of inhaled corticosteroids, long-acting antimuscarinic or long-acting $\beta 2$-agonists. GOLD: Global Initiative for Chronic Obstructive Lung Disease.

Asian patients with chronic respiratory diseases. It is plausible that, given differences in genetics, environment and lifestyle, the airway microbiome in Asia may differ from that observed in Western cohorts. In the COMPASS digital and biomarker subgroup, in addition to in-depth clinical and biomarker profiling we will conduct a detailed analysis using induced or spontaneous sputum samples to analyse the bacterial, viral and fungal components of the airway microbiome. Sputum samples are more easily obtainable than bronchoalveolar lavage samples, and studies have shown that sputum has utility in assessing the abundance of microbiota and that sputum microbiome alterations correlate with clinical outcomes [27]. These sputum samples will be collected at baseline, at the last visit (Month 30 or early withdrawal visit) and following a visit for an exacerbation. This will be one of the largest studies of the airway microbiome to date.

\section{Sample size considerations}

This study is exploratory with no formal pre-specified hypotheses associated with the study objectives. Confidence intervals will accompany all effects estimates. Sample size justification is based on precision (half width of confidence interval) for the difference between the rates of decline in $\mathrm{FEV}_{1}$ of Global Initiative for Chronic Obstructive Lung Disease (GOLD) 1 and GOLD 2 subjects over 2.5 years. Under the foregoing assumptions, with 95\% confidence interval and a 25\% drop out, 700 subjects for each group 


\section{TABLE 1 Inclusion criteria}

\begin{tabular}{|c|c|c|c|}
\hline & $\begin{array}{l}\text { COPD } \\
\text { participants }\end{array}$ & $\begin{array}{l}\text { Chronic bronchitis } \\
\text { participants }\end{array}$ & $\begin{array}{l}\text { Healthy } \\
\text { participants }\end{array}$ \\
\hline \multicolumn{4}{|l|}{ Inclusion criteria (all the following criteria apply) } \\
\hline Aged $50-80$ years inclusive & $\checkmark$ & $\checkmark$ & $\checkmark$ \\
\hline Baseline (post-bronchodilator) $\mathrm{FEV}_{1} / \mathrm{FVC}$ ratio & $<70 \%$ & $\geqslant 70 \%$ & $\geqslant 70 \%$ \\
\hline CAT score $<10$ & & & $\checkmark$ \\
\hline $\mathrm{BMI}<35$ (for CT scanning reasons) & $\checkmark$ & $\checkmark$ & $\checkmark$ \\
\hline $\begin{array}{l}\text { Chronic mucus hypersecretion ( } \geqslant 3 \text { months of } \\
\text { cough and phlegm in a year in the past } 2 \text { years) }\end{array}$ & & $\checkmark$ & \\
\hline $\begin{array}{l}\text { No exacerbations for } \geqslant 1 \text { month prior to } \\
\text { recruitment }\end{array}$ & $\checkmark$ & $\checkmark$ & \\
\hline $\begin{array}{l}\text { Ever-smoker (lifetime exposure of } \geqslant 1 \text { pack-year) or } \\
\text { never-smoker (lifetime exposure of }<1 \text { pack-year) } \\
\text { (for healthy participants, ever-smokers and passive } \\
\text { smokers are not eligible) }\end{array}$ & $\checkmark$ & $\checkmark$ & $\checkmark$ \\
\hline \multicolumn{4}{|l|}{ Additional criteria for sub-cohort of COPD participants } \\
\hline $\begin{array}{l}150 \text { COPD participants with } \geqslant 1 \text { moderate or severe } \\
\text { exacerbation event within the past } 2 \text { years }\end{array}$ & $\checkmark$ & N/A & N/A \\
\hline $\begin{array}{l}60 \text { COPD participants without inhaler maintenance } \\
\text { treatment history for } \geqslant 12 \text { months prior to the } \\
\text { entry of the study }\end{array}$ & $\checkmark$ & $\mathrm{N} / \mathrm{A}$ & $\mathrm{N} / \mathrm{A}$ \\
\hline
\end{tabular}

(GOLD 1 and GOLD 2) should be enrolled to maintain a precision (half width of confidence interval) of no more than $15 \mathrm{~mL} \cdot$ year $^{-1}$ for the difference between the rates of decline in $\mathrm{FEV}_{1}$ of GOLD 1 to GOLD 2 subjects. Within each GOLD group and the chronic bronchitis group, a recruitment target of 15-30\% of never-smokers has been set.

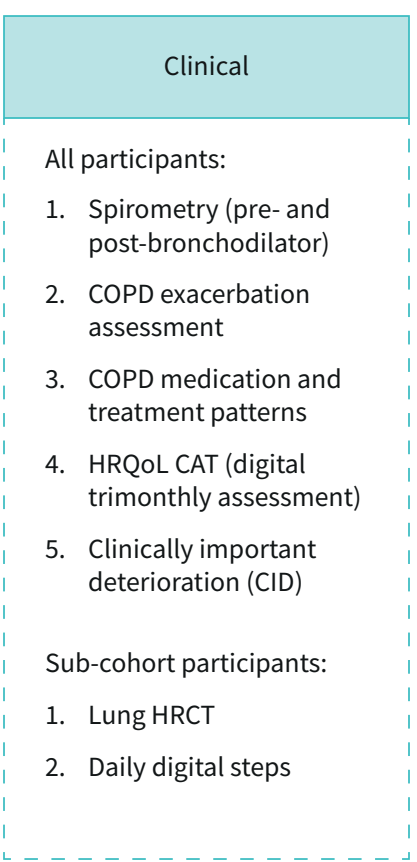

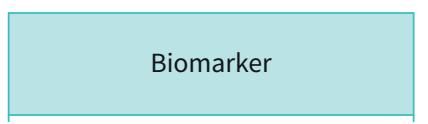

All participants:

1. Blood test: plasma fibrinogen and hsCRP, total and differential blood cell count

Sub-cohort participants:

1. Blood biomarkers (including serum SRAGE, CC16, IP-10, HbA1c)

2. Sputum microbiome bacteriome, virome, mycobiome

3. Sputum cytology total and differential leukocyte counts
Health economics and outcomes

All participants:

1. All-cause mortality

2. Patient-reported outcomes: CAT SGRQ-C, mMRC and Capture

3. Healthcare resource utilisation

Sub-cohort participants:

1. Exacerbations of Chronic Pulmonary Disease Tool (EXACT)

2. Evaluating Respiratory Symptoms in COPD (E-RS: COPD)

3. Digital daily diary

FIGURE 2 COMPASS Study end-points overview. HRQoL: health-related quality of life; CAT: COPD Assessment Test; HRCT: high-resolution computed tomography; hsCRP: high-sensitivity C-reactive protein; SGRQ-C: St George's Respiratory Questionnaire, COPD-specific version; mMRC: Modified Medical Research Council Dyspnoea Scale. 


\section{Study organisation}

The COMPASS study is guided by a steering committee, consisting of academic senior investigators and representatives from the study sponsor (GlaxoSmithKline) (see figure 3). The recruiting sites are made up of a network of 25 Tier 3 university hospitals and 15 Tier 2 general hospitals. A number of Working Groups (WGs) will be established to cover different areas of research: e.g. imaging, microbiome, biomarker, spirometry, etc. Each will have a lead who may or may not come from the steering committee, and the membership will be comprised of senior and junior researchers. Overseas experts will be invited to join the WGs, as appropriate. Each WG will determine the scientific questions to be addressed in their area, specify the analyses to be done and write the papers. GlaxoSmithKline will provide operational and statistical support to the WGs. A hive structure is proposed in which the WGs also interact with each other to share insights and expertise.

\section{Discussion}

COMPASS has been designed to study the heterogeneity and complexity of COPD in China, building on disease understanding that has come from previous cohort studies in the West. It will support longitudinal analysis of a moderate sample size in terms of lung function and symptom progression measures which, together with a biomarker and digital subgroup, will provide in-depth data collected using HRCT, diary cards, activity monitors and biomarkers in blood and sputum. To ensure an adequate balance of different phenotypes, a key feature (and a significant recruitment challenge) is the establishment of specific recruitment targets for each clinical subgroup. The focus will be primarily on individuals with mildmoderate airflow limitation with specific targets for participants who may have different aetiologies and disease patterns; for example, never-smokers, individuals with mild airflow limitation but a history of exacerbations and treatment naïve individuals who had not been taking regular maintenance treatment before entry into the study.

COMPASS will allow us to test whether the phenotypes and endotypes seen in China follow patterns identified in Western cohorts. Whilst it is likely that no new types of COPD will be identified, their contribution may differ due to factors such as genetics, prior chest disease and environmental factors. The study will also focus recruitment on more defined populations than its predecessors, particularly patients with mild airflow limitation and never-smokers, so it may include unique features that will extend our understanding of COPD more generally. As aforementioned, COMPASS will recruit a high proportion of participants (70\%) with milder COPD (GOLD 1-2) at baseline. These subjects will provide the

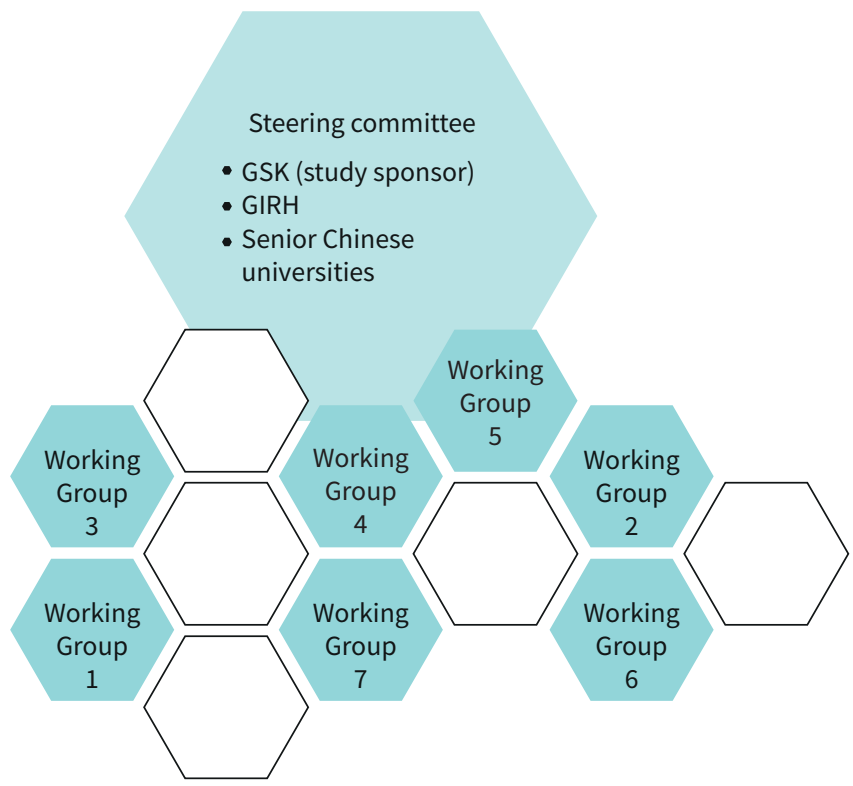

FIGURE 3 COMPASS Research network and the organisation of steering. The steering committee is made up of experts from GlaxoSmithKline (GSK), Guangzhou Institute of Respiratory Health (GIRH) and other senior Chinese universities. The Working Groups (WGs) will each have their own lead who will work closely with the steering committee. The number of WGs is illustrative. A hive structure is planned in which WGs will interact with each other as well as with the steering committee. 
opportunity to investigate the lung function trajectories and disease progression patterns in mild-moderate COPD yielding insights and evidence for early intervention. Unlike the GOLD 3 and 4 COPD population, the GOLD 1 and 2 patients are a population that has not been well studied global wide due to the difficulties in identification and recruitment. More than 70\% of patients with mild COPD have a low level of clinical symptoms which do not get the attention of the patients themselves or their doctors. Effective diagnosis in this population depends on the identification of risk factors and case-finding for spirometry, so few of them get detected and start intervention at a mild stage. However, lung function declines rapidly in these patients, and damage to the small airways is evident [28-30]. A previous study found that lung function of patients with GOLD Grade 2 declines more rapidly than patients with Grades 3 and 4, but few data are available for Grade 1 disease [31]. The recruitment to COMPASS is being carried out in a range of hospital types, not just university hospitals, because in China, patients are free to select their hospital and may triage themselves to higher grade hospitals if they have more symptoms. A further factor that should support COMPASS recruiting milder patients is that in recent years, with continuous investment and increasing attention from the Chinese government, the management of COPD in China is shifting from disease treatment to a focus on public health. This should create a good environment for the recruitment of COPD patients at an early stage in their disease, and it is a hope that COMPASS will help inform an early intervention strategy for COPD in China.

To understand the relationship between patients with mild airflow limitation and those with chronic bronchitis but normal spirometry, recruitment will include a target for people with chronic bronchitis including those who have been never-smokers. The subgroup of COPD participants who had not previously received maintenance treatment is primarily to allow comparison of their airway micro-, viraland myco-biome with participants with similar degrees of airflow limitation who have received treatment such as long-acting bronchodilators and inhaled corticosteroids. This should also provide the opportunity to study changes of airway microbiota in participants who may have maintenance treatment initiated during the course of the study. From a clinical perspective, a particular feature of the protocol for the biomarker and digital subgroup is the investigations to be carried out during an acute exacerbation that will include HRCT, biomarker and airway microbiome analyses.

In addition to the clinical and scientific investigations, COMPASS will also investigate the feasibility of aggregating different types of digitally acquired data including electronic health records, electronic clinical outcome assessments and physical activity data through direct digital data capture and integration. In part, the purpose of this is to develop systems of collecting data that permit clinical trials of treatment efficacy and effectiveness in a setting that is closer to a real-world setting and minimise the influences and biases that can come from close or regular contact with healthcare professionals that is not reflected in routine care.

As with previous similar cohort studies (and where possible using similar methodology), COMPASS is designed to provide a cross-sectional comparison between well-defined groups of COPD patients and measure disease progression. It will also integrate these data with details of healthcare resource use, including treatment. The characterisation of defined phenotypes and endotypes ("treatable traits") and their stability in mild-moderate patients over time will be important for the development of new treatments and the management of patients in the future. In that respect, a key feature of COMPASS is use of the Exacerbations of Chronic Pulmonary Disease Tool (EXACT) diary to detect unreported exacerbations and determine their impact on the clinical status or accelerated disease progression.

The study is conducted mainly in the Guangdong province of China. A key reason for this was the availability of a diverse patient population to be recruited. Whilst confining the study primarily to Guangdong may appear to be a limitation since the findings may not represent the broader Chinese patient population, this is not a major concern. The total population of Guangdong was 104 million in the 2010 census $(7.9 \%$ of the total population of China), of which 31 million were migrants from other areas of China who had spent at least 6 months of the previous year in Guangdong. Furthermore, this province has been growing fast in the past 15 years to become one of the biggest regions in China with a large population of younger people.

It is important to recognise that this study is not designed to determine the epidemiology of different COPD phenotypes in China. That is clear from the targeted recruitment strategy. Its purpose is to build on previous cohort studies to understand the patterns of disease and disease progression in patients in an Asian setting. Its importance is not limited to Asia, however, because understanding the heterogeneity and complexity of COPD in different environments and in patients with different backgrounds will lead to a greater understanding of the disease more generally and give greater insight into reasons why it is expressed differently in different patients. 
In summary, COMPASS will establish a rich set of clinical and biological data on a relatively large cohort of well-characterised individuals with COPD in China of varying degrees of severity and different aetiologies, including patients who never smoked tobacco and may have COPD related to other factors such as environmental exposure to pollution including biomass smoke. The study will provide unique and detailed insight into personalised information about the pulmonary and extrapulmonary aspects of disease progression in Chinese patients, especially those with mild-moderate airflow limitation. It holds great promise to address the heterogeneity and complexity of multiple components in COPD.

The members of the COMPASS Steering Committee at the time of developing this manuscript are: Nanshan Zhong (The First Affiliated Hospital of Guangzhou Medical University, Guangzhou, China); Jinping Zheng (The First Affiliated Hospital of Guangzhou Medical University, Guangzhou, China); Rongchang Chen (First Affiliated Hospital of South University of Science and Technology of China, Shenzhen, China); Qianli Ma (The North Kuanren General Hospital, Chongqing, China); Yongchang Sun (Peking University Third Hospital, Beijing, China); Fuqiang Wen (West China Hospital of Sichuan University, Chengdu, China); Paul Jones (GlaxoSmithKline, Brentford, UK); Chris Compton (GlaxoSmithKline, Brentford, UK); Bruce E. Miller (GlaxoSmithKline, Collegeville, PA, USA); Julie Yates (GlaxoSmithKline, Research Triangle Park, NC, USA); Beulah Ji (GlaxoSmithKline, Research and Development, Shanghai, China); Jie Song (GlaxoSmithKline, Research and Development, Shanghai, China).

Author contributions: Nanshan Zhong, Rongchang Chen, Qianli Ma, Yongchang Sun, Fuqiang Wen, Ruth Tal-Singer, Bruce E. Miller, Julie Yates, Jie Song, Chris Compton, Beulah Ji, Li Wu, Yang Yang, Paul Jones and Jinping Zheng were involved in the study design and/or conception. Zhenyi Liang, Nanshan Zhong, Jinping Zheng and Rongchang Chen were involved in the acquisition of data. All authors interpreted data, wrote and reviewed the manuscript, and gave final approval of the manuscript.

This study is registered at www.clinicaltrials.gov with identifier number NCT04853225.

Data availability: Data will be made available when the study has completed through the Clinical Study Data Request website: ClinicalStudyDataRequest.com

Conflict of interest: Z. Liang reports support for the present manuscript from GlaxoSmithKline, which funded the study (Study 208630) and National Key R\&D Program of China, which supported the study (2018YFC1311900, 2017YFC1310600). The author reports other financial or nonfinancial interests as follows: key coordinator between GlaxoSmithKline and COMPASS investigators, and clinical expert for the First Affiliated Hospital of Guangzhou Medical University. N. Zhong reports support for the present manuscript from GlaxoSmithKline, which funded the study (Study 208630) and National Key R\&D Program of China, which supported the study (2018YFC1311900, 2017YFC1310600). The author reports other financial or non-financial interests as follows: member of the COMPASS steering committee for GlaxoSmithKline and a medical expert for the First Affiliated Hospital of Guangzhou Medical University. R. Chen reports support for the present manuscript from GlaxoSmithKline, which funded the study (Study 208630) and National Key R\&D Program of China, which supported the study (2018YFC1311900 and 2017YFC1310600). The author reports other financial or nonfinancial interests as follows: member of the COMPASS steering committee for GlaxoSmithKline and a medical expert for the First Affiliated Hospital of South University of Science and Technology of China. Q. Ma reports support for the present manuscript from GlaxoSmithKline, which funded the study (Study 208630) and National Key R\&D Program of China, which supported the study (2018YFC1311900 and 2017YFC1310600). The author reports other financial or nonfinancial interests as follows: member of the COMPASS steering committee for GlaxoSmithKline and a medical expert for the North Kuanren General Hospital. Y. Sun reports support for the present manuscript from GlaxoSmithKline, which funded the study (Study 208630) and National Key R\&D Program of China, which supported the study (2018YFC1311900 and 2017YFC1310600). The author reports other financial or nonfinancial interests as follows: member of the COMPASS steering committee for GlaxoSmithKline and a medical expert for Peking University Third Hospital. F. Wen reports support for the present manuscript from GlaxoSmithKline, which funded the study (Study 208630) and National Key R\&D Program of China, which supported the study (2018YFC1311900 and 2017YFC1310600). The author reports other financial or nonfinancial interests as follows: member of COMPASS steering committee for GlaxoSmithKline and medical expert for West China Hospital of Sichuan University. R. Tal-Singer reports support for the present manuscript from GlaxoSmithKline, which funded the study (Study 208630) and National Key R\&D Program of China, which supported the study (2018YFC1311900 and 2017YFC1310600). The author also reports that they currently hold stock of GlaxoSmithKline and a former employee of GlaxoSmithKline. B. Miller reports support for the present manuscript from GlaxoSmithKline, which funded the study (Study 208630) and National Key R\&D Program of China, which supported the study (2018YFC1311900 and 2017YFC1310600). The author also reports that they currently hold stock of GlaxoSmithKline and a former employee of GlaxoSmithKline and member of COMPASS steering committee for GlaxoSmithKline. J. Yates reports support for the present manuscript from 
GlaxoSmithKline, which funded the study (Study 208630) and National Key R\&D Program of China, which supported the study (2018YFC1311900 and 2017YFC1310600). The author also reports that they currently hold stock of GlaxoSmithKline and a former employee of GlaxoSmithKline and member of COMPASS steering committee for GlaxoSmithKline. J. Song reports support for the present manuscript from GlaxoSmithKline, which funded the study (Study 208630) and National Key R\&D Program of China, which supported the study (2018YFC1311900 and 2017YFC1310600). The author also reports that they are currently an employee of GlaxoSmithKline and member of the COMPASS steering committee for GlaxoSmithKline. C. Compton reports support for the present manuscript from GlaxoSmithKline, which funded the study (Study 208630) and National Key R\&D Program of China, which supported the study (2018YFC1311900 and 2017YFC1310600). The author also reports that they currently hold stock of GlaxoSmithKline and a former employee of GlaxoSmithKline and member of COMPASS steering committee for GlaxoSmithKline. B. Ji reports support for the present manuscript from GlaxoSmithKline, which funded the study (Study 208630) and National Key R\&D Program of China, which supported the study (2018YFC1311900 and 2017YFC1310600). The author also reports that they currently hold stock of GlaxoSmithKline and are an employee of GlaxoSmithKline and member of COMPASS steering committee. L. Wu reports support for the present manuscript from GlaxoSmithKline, which funded the study (Study 208630) and National Key R\&D Program of China, which supported the study (2018YFC1311900 and 2017YFC1310600). The author also reports that they currently employed by GlaxoSmithKline. Y. Yang reports support for the present manuscript from GlaxoSmithKline, which funded the study (Study 208630) and National Key R\&D Program of China, which supported the study (2018YFC1311900 and 2017YFC1310600). The author also reports that they are currently an employee of GlaxoSmithKline. P. Jones reports support for the present manuscript from GlaxoSmithKline, which funded the study (Study 208630) and National Key R\&D Program of China, which supported the study (2018YFC1311900 and 2017YFC1310600). The author also reports that they are currently an employee of GlaxoSmithKline and member of the COMPASS steering committee for GlaxoSmithKline. The author currently holds stock in GlaxoSmithKline. Jinping Zheng reports support for the present manuscript from GlaxoSmithKline, which funded the study (Study 208630) and National Key R\&D Program of China, which supported the study (2018YFC1311900 and 2017YFC1310600). The author reports other financial or nonfinancial interests as follows: member of COMPASS steering committee for GlaxoSmithKline and medical expert for the First Affiliated Hospital of Guangzhou Medical University.

Support statement: This study was supported by GSK and the National Key R\&D Program of China grants 2018YFC1311900 and 2017YFC1310600. Funding information for this article has been deposited with the Crossref Funder Registry.

\section{References}

1 World Health Organization. Chronic Obstructive Pulmonary Disease (COPD). https://www.who.int/news-room/ fact-sheets/detail/chronic-obstructive-pulmonary-disease-(copd) Date last accessed: 12 August 2021.

2 Zhong N, Wang C, Yao W, et al. Prevalence of chronic obstructive pulmonary disease in China: a large, population-based survey. Am J Respir Crit Care Med 2007; 176: 753-760.

3 Wang C, Xu J, Yang L, et al. Prevalence and risk factors of chronic obstructive pulmonary disease in China (the China Pulmonary Health [CPH] study): a national cross-sectional study. Lancet 2018; 391: 1706-1717.

4 Jones PW, Brusselle G, Dal Negro RW, et al. Properties of the COPD assessment test in a cross-sectional European study. Eur Respir J 2011; 38: 29-35.

5 Agusti A, Celli B, Faner R. What does endotyping mean for treatment in chronic obstructive pulmonary disease? Lancet 2017; 390: 980-987.

6 Hiles SA, Gibson PG, Agusti A, et al. Treatable traits that predict health status and treatment response in airway disease. J Allergy Clin Immunol Pract 2021; 9: 1255-1264.e2.

7 van Dijk M, Gan CT, Koster TD, et al. Treatment of severe stable COPD: the multidimensional approach of treatable traits. ERJ Open Res 2020; 6: 00322-2019.

8 Vestbo J, Anderson W, Coxson HO, et al. Evaluation of COPD longitudinally to identify predictive surrogate end-points (ECLIPSE). Eur Respir J 2008; 31: 869-873.

9 Regan EA, Hokanson JE, Murphy JR, et al. Genetic epidemiology of COPD (COPDGene) study design. COPD 2010; 7: 32-43.

10 Couper D, LaVange LM, Han M, et al. Design of the subpopulations and intermediate outcomes in COPD study (SPIROMICS). Thorax 2014; 69: 491-494.

11 Reddel HK, Gerhardsson DVM, Agusti A, et al. Prospective observational study in patients with obstructive lung disease: NOVELTY design. ERJ Open Res 2019; 5: 00036-2018.

12 Beran D, Zar HJ, Perrin C, et al. Burden of asthma and chronic obstructive pulmonary disease and access to essential medicines in low-income and middle-income countries. Lancet Respir Med 2015; 3: 159-170.

13 Agusti A, Hogg JC. Update on the pathogenesis of chronic obstructive pulmonary disease. N Engl J Med 2019; 381: $1248-1256$ 

Western COPD patients in a prospective, international, multicenter study. Int J Chron Obstruct Pulmon Dis 2019; 14: 1595-1601.

15 Lee SY, Mac AM, Fam KD, et al. Airway microbiome composition correlates with lung function and arterial stiffness in an age-dependent manner. PLOS One 2019; 14: e225636.

16 GBD 2015 Chronic Respiratory Disease Collaborators. Global, regional, and national deaths, prevalence, disability-adjusted life years, and years lived with disability for chronic obstructive pulmonary disease and asthma, 1990-2015: a systematic analysis for the Global Burden of Disease Study 2015. Lancet Respir Med 2017; 5: 691-706.

17 Gordon SB, Bruce NG, Grigg J, et al. Respiratory risks from household air pollution in low and middle income countries. Lancet Respir Med 2014; 2: 823-860.

18 Zhong $\mathrm{N}$, Moon HS, Lee $\mathrm{KH}$, et al. TIOtropium safety and performance in respimat((R)) (TIOSPIR(TM)): analysis of Asian cohort of COPD patients. Respirology 2016; 21: 1397-1403.

19 Wise RA, Anzueto A, Calverley P, et al. The tiotropium safety and performance in respimat trial (TIOSPIR), a large scale, randomized, controlled, parallel-group trial-design and rationale. Respir Res 2013; 14: 40.

20 Buist AS, McBurnie MA, Vollmer WM, et al. International variation in the prevalence of COPD (the BOLD Study): a population-based prevalence study. Lancet 2007; 370: 741-750.

21 Tan WC, Bourbeau J, Hernandez P, et al. Exacerbation-like respiratory symptoms in individuals without chronic obstructive pulmonary disease: results from a population-based study. Thorax 2014; 69: 709-717.

22 Miller BE, Tal-Singer R, Rennard SI, et al. Plasma fibrinogen qualification as a drug development tool in chronic obstructive pulmonary disease. Perspective of the chronic obstructive pulmonary disease biomarker qualification consortium. Am J Respir Crit Care Med 2016; 193: 607-613.

23 Faner R, Sibila O, Agusti A, et al. The microbiome in respiratory medicine: current challenges and future perspectives. Eur Respir J 2017; 49: 1602086.

24 Mayhew D, Devos N, Lambert C, et al. Longitudinal profiling of the lung microbiome in the AERIS study demonstrates repeatability of bacterial and eosinophilic COPD exacerbations. Thorax 2018; 73: 422-430.

25 Wang Z, Singh R, Miller BE, et al. Sputum microbiome temporal variability and dysbiosis in chronic obstructive pulmonary disease exacerbations: an analysis of the COPDMAP study. Thorax 2018; 73: 331-338.

26 Dicker AJ, Huang J, Lonergan $\mathrm{M}$, et al. The sputum microbiome, airway inflammation, and mortality in chronic obstructive pulmonary disease. J Allergy Clin Immunol 2021; 147: 158-167.

27 Ditz B, Christenson S, Rossen J, et al. Sputum microbiome profiling in COPD: beyond singular pathogen detection. Thorax 2020; 75: 338-344.

28 Zhong NS, Zeng GQ. Prevention and treatment of chronic respiratory diseases in China. Chronic Dis Transl Med 2019; 5: 209-213.

29 Koo HK, Vasilescu DM, Booth S, et al. Small airways disease in mild and moderate chronic obstructive pulmonary disease: a cross-sectional study. Lancet Respir Med 2018; 6: 591-602.

30 Russell DW, Wells JM. COPD ground zero: small airways rather than alveoli as the initial site of injury. Lancet Respir Med 2018; 6: 568-569.

31 Tantucci C, Modina D. Lung function decline in COPD. Int J Chron Obstruct Pulmon Dis 2012; 7: 95-99. 